\title{
Eddy current damping of a moving domain wall: Beyond the quasistatic approximation
}

\author{
Francesca Colaiori, ${ }^{1}$ Gianfranco Durin, ${ }^{2}$ and Stefano Zapperi ${ }^{1,3}$ \\ ${ }^{1}$ CNR-INFM, SMC, Dipartimento di Fisica, Università di Roma "La Sapienza”, Piazzale A. Moro 2, 00185 Roma, Italy \\ ${ }^{2}$ INRIM, Strada delle Cacce 91, 10137 Torino, Italy \\ ${ }^{3}$ ISI Foundation, Viale S. Severo 65, 10133 Torino, Italy
}

(Received 12 June 2007; revised manuscript received 14 September 2007; published 19 December 2007)

\begin{abstract}
In conducting ferromagnetic materials, a moving domain wall induces eddy currents in the sample, which give rise to an effective retarding pressure on the domain wall. We show here that the pressure is not just proportional to the instantaneous velocity of the wall, as often assumed in domain wall models, but depends on the history of the motion. We calculate the retarding pressure by solving the Maxwell equations for the field generated by the eddy currents and show how its effect can be accounted for by associating a negative effective mass to the magnetic wall. We analyze the dependence of this effect on the sample geometry and discuss the implications for the Barkhausen noise measurements.
\end{abstract}

DOI: 10.1103/PhysRevB.76.224416

PACS number(s): 75.60.Ch, 75.60.Ej

\section{INTRODUCTION}

Soft magnetic materials subject to a slowly changing external magnetic field, respond with a jerky motion of the domain walls, known as the Barkhausen noise. ${ }^{1,2}$ Many universal properties of this noise have been identified and are correctly reproduced by theoretical models, confirming that, in analogy to critical phenomena, the statistical features of the signal only depend on general properties of the physical mechanism, which govern the magnetization reversal process, while they are independent of the microscopic details of the particular system. ${ }^{3-5}$

The average shape of the pulse, being a nonscalar quantity, has been successfully identified as a powerful tool to characterize the universal properties of crackling systems: pulses of different durations, once properly rescaled, are expected to collapse onto a universal function. ${ }^{6}$ In the case of the Barkhausen noise, pulses from experimental data do approximately collapse on the same curve, $;^{7,8}$ however, this curve shows a clear leftward asymmetry, while models that very accurately reproduce most of the other universal quantities predict a symmetric shape. ${ }^{9}$

As shown in Ref. 10, the asymmetry in the Barkhausen pulses is due to the noninstantaneous response of the eddy current field to the domain wall displacement. The Barkhausen noise models usually assume this response to be instantaneous and thus do not capture this asymmetry. Since eddy currents take a finite time to set up and also they persist for a finite time after the corresponding wall displacement, the pressure on the moving domain wall is not strictly proportional to the instantaneous velocity of the wall but depends on the history of the motion. The delay has a characteristic time scale, and therefore, its effect is more evident on avalanches of comparable duration, and disappears on very large ones, where the separation of time scales is such that the response of the field can be assumed to be instantaneous, and strict universality is recovered. The first order correction to the instantaneous response approximation can be accounted for by associating a negative effective mass to the wall in the equation of motion. ${ }^{10}$

In this paper, we report a detailed calculation of the retarding pressure starting from the Maxwell equations and obtain the negative effective mass as a first order correction to the quasistatic approximation. Our approach is similar to the one of Bishop. ${ }^{11}$ The resulting nonlocal damping was previously employed in a domain wall dynamics model in Ref. 10, where its effect on the Barkhausen pulse shape was studied and compared with experimental data. Here, we analyze the role of sample geometry on the eddy current propagation and provide expression for the damping term and the effective mass as a function of the sample aspect ratio.

The paper is organized as follows. In Sec. II, we solve the Maxwell equations for the eddy currents in a conducting sample with a moving domain wall. In Sec. III, we compute the resulting pressure on the domain wall. In Sec. IV, we derive the first order correction to the pressure. In Sec. V, we discuss the role of the sample geometry, and in Sec. VI, we conclude. Finally, two Appendixes report the details of some series summation used in the paper.

\section{EDDY CURRENT FIELD FROM THE MAXWELL EQUATIONS}

Consider a sample with dimensions $x \in[-a / 2, a / 2], y$ $\in[-b / 2, b / 2]$, and infinite in the $z$ direction, divided in two magnetic domains by a rigid domain wall on the $y z$ plane, moving from position $x=0$, as in Fig. 1. The displacement of a magnetic wall in a conducting medium induces a flow of eddy currents that generate a magnetic field, which, in the geometry indicated in Fig. 1, is parallel to the $z$ axis,

$$
\vec{H}_{e}=H_{e}(x, y, t) \hat{z} \text {. }
$$

The magnetic field obeys the Maxwell equation,

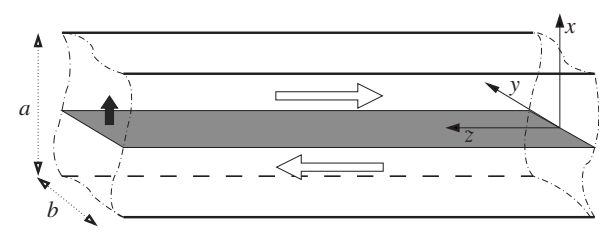

FIG. 1. Horizontal arrows indicate the directions of the magnetization in the two domains. The black vertical arrow indicates the direction of motion of the wall. 


$$
\nabla^{2} H_{e}=\epsilon \partial_{t} H_{e},
$$

with $\epsilon=\sigma \mu$, where $\sigma$ and $\mu$ are the electric conductivity and the magnetic permeability of the medium. ${ }^{12}$ In Eq. (2), the displacement currents are neglected with respect to the Ohmic currents. This equation is usually solved in the quasistatic approximation, where $\epsilon$ is negligible within domains, and the equation reduces to $\nabla^{2} H_{e}=0$. Eq. (2) is a diffusion equation: there is a finite time delay between the wall displacement and the establishment of the eddy currents. Given that the typical time scale for diffusion is proportional to $\epsilon$, the quasistatic approximation $\epsilon=0$ corresponds to assuming an instantaneous response of the field.

To take into account dynamical effects from eddy currents in the domain wall propagation, we need to solve Eq. (2), with the appropriate boundary condition $H_{e}=0$ on the sample surface. The discontinuity across the wall is regulated by the Faraday condition $\partial_{x} H_{e}\left(0^{+}, y, t\right)-\partial_{x} H_{e}\left(0^{-}, y, t\right)=2 \sigma I v(t)$, where $I$ is the saturation magnetization and $v(t)$ is the velocity of the wall.

Let us expand $H_{e}$ in its Fourier components,

$$
H_{e}(x, y, t)=\frac{1}{\sqrt{2 \pi}} \int_{-\infty}^{\infty} d \omega F(x, y, \omega) e^{i \omega t} .
$$

Each component has to satisfy

$$
\nabla F(x, y, \omega)=r^{2} F(x, y, \omega),
$$

with $r^{2}=i \omega \epsilon$. The magnetic field has to be zero on the sample boundary, which implies the conditions $F(x, \pm b / 2, \omega)$ $=F( \pm a / 2, y, \omega)=0$. Moreover, the Faraday condition around the wall imposes $\partial_{x} F\left(0^{+}, y, \omega\right)-\partial_{x} F\left(0^{-}, y, \omega\right)=2 \sigma I \hat{v}(\omega)$, where $\hat{v}$ is the Fourier transform of the velocity of the wall $v(t)$. The solution is of the form

$$
F(x, y, \omega)=\sum_{n=0}^{\infty} A_{n}(x, \omega) \cos \left(\lambda_{n} y\right),
$$

where $A_{n}$ satisfies

$$
\partial_{x}^{2} A_{n}(x, \omega)=\Lambda_{n}^{2} A_{n}(x, \omega),
$$

with $\Lambda_{n}^{2}=\lambda^{2}+r^{2}$, to be solved separately for $x>0$ and $x<0$. The condition on the sample boundary in the $y$ direction implies $\cos \left( \pm b / 2 \lambda_{n}\right)=0$ which fixes $\lambda_{n}=(2 n+1) \pi / b$. The condition on the other boundary is satisfied by choosing

$$
A_{n}(x, \omega)=C_{n}(\omega) \sinh \left[\Lambda_{n}(|x|-a / 2)\right],
$$

so that

$$
F(x, y, \omega)=\sum_{n=0}^{\infty} C_{n}(\omega) \sinh \left[\Lambda_{n}(\omega)(|x|-a / 2)\right] \cos \left(\lambda_{n} y\right) .
$$

The functions $C_{n}(\omega)$ are fixed by the Faraday condition,

$$
\begin{aligned}
\partial_{x} F\left(0^{+}, y, \omega\right) & =-\partial_{x} F\left(0^{-}, y, \omega\right) \\
& =\sum_{n=0}^{\infty} C_{n}(\omega) \Lambda_{n}(\omega) \cosh \left[\Lambda_{n}(\omega) a / 2\right] \cos \left(\lambda_{n} y\right) \\
& =\sigma I \hat{v}(\omega) .
\end{aligned}
$$

Multiplying by $\cos \left(\lambda_{m} y\right)$, integrating in $[-b / 2, b / 2]$, and using the orthogonality relations $\int_{-b / 2}^{b / 2} d y \cos \left(\lambda_{n} y\right) \cos \left(\lambda_{m} y\right)$ $=\delta_{n, m} b / 2$ and $\int_{-b / 2}^{b / 2} d y \cos \left(\lambda_{m} y\right)=(-1)^{m} 2 / \lambda_{m}$, we get

$$
C_{n}(\omega)=(-1)^{n} \frac{4 \sigma I}{b} \frac{1}{\lambda_{n} \Lambda_{n}(\omega) \cosh \left[\Lambda_{n}(\omega) a / 2\right]} \hat{v}(\omega),
$$

so that finally,

$$
\begin{aligned}
F(x, y, \omega)= & \frac{4 \sigma I}{b} \sum_{n=0}^{\infty}(-1)^{n} \frac{\sinh \left[\Lambda_{n}(\omega)(|x|-a / 2)\right]}{\lambda_{n} \Lambda_{n}(\omega) \cosh \left[\Lambda_{n}(\omega) a / 2\right]} \\
& \times \cos \left(\lambda_{n} y\right) \hat{v}(\omega) .
\end{aligned}
$$

In the next section, we will need the value of the field at $x=0$, where $F$ simplifies to

$$
F(0, y, \omega)=\frac{4 \sigma I}{b} \sum_{n=0}^{\infty}(-1)^{n} \frac{\tanh \left[\Lambda_{n}(\omega) a / 2\right]}{\lambda_{n} \Lambda_{n}(\omega)} \cos \left(\lambda_{n} y\right) \hat{v}(\omega) .
$$

\section{EDDY CURRENT PRESSURE ON THE WALL}

To understand how the eddy field affects the motion of the domain wall, we recall that the equation of motion of the wall is obtained by balancing the pressure $P$ due to the eddy field with the total pressure given by the effective force acting on the wall. The equation can be written as

$$
P=P_{e x t}+P_{d e m}+P_{p}
$$

where $P_{e x t}=2 I H_{e x t}$ is the pressure due to the external field $H_{e x t}, P_{\text {dem }}=-2 I \mathrm{kx}$ takes into account the stray contribution of the demagnetizing field, and $k$ takes into account the sample geometry, and $P_{p}=2 I H_{p}$ is a random contribution of local origin, with $H_{p}$ given by a random Brownian process in space. ${ }^{3}$ In the approximation of an instantaneous response of the field, the eddy pressure is proportional to the velocity of the wall, and the resulting motion is overdamped. We now calculate the left hand side of Eq. (13) taking into account the field relaxation.

Once the solution of the Maxwell equation with the appropriate boundary condition is given, the average eddy current pressure on the wall is obtained by integrating the magnetic field over $y$ at the wall position $x=0$,

$$
\begin{aligned}
P(t) & =\frac{2 I}{b} \int_{-b / 2}^{b / 2} d y H_{e}(0, y, t) \\
& =\frac{2 I}{b} \frac{1}{\sqrt{2 \pi}} \int_{-\infty}^{\infty} d \omega e^{i \omega t} \int_{-b / 2}^{b / 2} d y F_{\omega}(0, y),
\end{aligned}
$$

or, in terms of the Fourier transform,

$$
\hat{P}(\omega)=-\hat{v}(\omega) \hat{f}(\omega),
$$

with 


$$
\hat{f}(\omega)=\frac{16 I^{2} \sigma}{b^{2}} \sum_{n=0}^{\infty} \frac{\tanh \left[\Lambda_{n}(\omega) a / 2\right]}{\lambda_{n}^{2} \Lambda_{n}(\omega)} .
$$

In real space, the pressure at time $t$ is given by a convolution of velocities of the wall at all times prior to $t$ with the response function $f$,

$$
P(t)=\frac{1}{\sqrt{2 \pi}} \int_{-\infty}^{\infty} d s v(t-s) f(s) .
$$

To simplify the $\omega$ dependence in Eq. (16), we use the relation

$$
\tanh (z)=8 z \sum_{k=0}^{\infty} \frac{1}{\pi^{2}(2 k+1)^{2}+4 z^{2}},
$$

with $z=\Lambda_{n}(\omega) a / 2$, which gives

$$
\hat{f}(\omega)=\frac{64 a I^{2} \sigma}{b^{2}} \sum_{n, k=0}^{\infty} \frac{1}{\lambda_{n}^{2}\left[b^{2} \lambda_{k}^{2}+a^{2} \Lambda_{n}^{2}(\omega)\right]} .
$$

Replacing $\lambda_{n}$ and $\Lambda_{n}$ with their expressions, we get

$$
\hat{f}(\omega)=\frac{64 I^{2} \sigma}{a b^{2} \epsilon^{2}} \sum_{n, k=1}^{\infty} \frac{1}{n^{2} \omega_{b}\left(k^{2} \omega_{a}+n^{2} \omega_{b}+i \omega\right)},
$$

where $\bar{\Sigma}$ indicates a summation over odd numbers only, and $\omega_{a}=\tau_{a}^{-1}=\pi^{2} / \epsilon a^{2}$ and $\omega_{b}=\tau_{b}^{-1}=\pi^{2} / \epsilon b^{2}$. Given that $1 /\left(\omega_{0}+i \omega\right)$ has $\sqrt{2 \pi} \exp \left(-\omega_{0} t\right) \theta(t)$ as inverse Fourier transform [where $\theta(t)$ is the Heaviside theta function], it is easy to obtain from Eq. (20) the expression for $f$ in real space by antitransforming term to term in the double sum to get

$$
f(t)=\sqrt{2 \pi} \frac{64 I^{2} \sigma}{a b^{2} \epsilon^{2}} \sum_{n, k=1}^{\infty} \frac{1}{n^{2} \omega_{b}} e^{-\omega_{k, n} t} \theta(t),
$$

where $\omega_{k, n}=\tau_{k, n}^{-1}=k^{2} \omega_{a}+n^{2} \omega_{b}$. The response function results to be the sum of simple exponential relaxations, with different relaxation times. The largest and therefore most relevant relaxation time is $\tau_{0,0}=\frac{\sigma \mu}{\pi^{2}}\left(\frac{1}{a^{2}}+\frac{1}{b^{2}}\right)^{-1}$.

Form (21) of the response function gives, through convolution (14), the explicit solution for the retarded pressure on the wall.

\section{FIRST ORDER CORRECTION: DAMPING COEFFICIENT AND NEGATIVE EFFECTIVE MASS}

Given the full solution of the response function, we now want to calculate the first order correction to the quasistatic approximation $\epsilon=0$ in the retarded pressure. In order to do this, let us replace expression (21) in convolution (14) and exchange the sum with the integral,

$$
P(t)=\frac{64 I^{2} \sigma}{a b^{2} \epsilon^{2}} \overline{\sum_{n, k=1}^{\infty}} \frac{1}{n^{2} \omega_{b}} \int_{0}^{\infty} d s v(t-s) e^{-\omega_{k, n^{s}}},
$$

where the Heaviside function was eliminated by restricting the domain of integration. For small relaxation times (note that $\tau_{k, n}<\tau_{0,0}$ for every $k$ and $n$ ), the exponential functions in the integral decay very fast around $s=0$, so that the velocities that sensibly contribute to the convolutions are only those at time very close to $t$. We may thus expand $v(t-s) \simeq v(t)-s v^{\prime}(t)$ around $s=t$ and perform the integrals. The term proportional to $v(t)$ corresponds the usual instantaneous contribution, while the term proportional to $v^{\prime}(t)$ gives rise to the first order correction to the quasi static approximation,

$$
P(t) \simeq \frac{64 I^{2} \sigma}{a b^{2} \epsilon^{2}} \overline{\sum_{n, k=1}^{\infty}} \frac{1}{n^{2} \omega_{b}}\left(\frac{v(t)}{\omega_{k, n}}-\frac{v^{\prime}(t)}{\omega_{k, n}^{2}}\right) .
$$

Replacing the frequencies with their expression,

$$
P(t) \simeq \frac{64 I^{2} \sigma}{\pi^{4}} \frac{b^{2}}{a}\left[v(t) \Sigma_{1}(b / a)-v^{\prime}(t) \tau_{b} \Sigma_{2}(b / a)\right],
$$

where $\Sigma_{1}(\alpha)=\bar{\Sigma}_{n, k} \frac{1}{n^{2}} \frac{1}{n^{2}+\alpha^{2} k^{2}}$ and $\Sigma_{2}(\alpha)=\bar{\Sigma}_{n, k} \frac{1}{n^{2}} \frac{1}{\left(n^{2}+\alpha^{2} k^{2}\right)^{2}}$. Equation (24) allows us to identify a damping coefficient,

$$
\Gamma=\frac{64 I^{2} \sigma b^{2}}{a \pi^{4}} \Sigma_{1}(b / a),
$$

and an effective mass per unit wall area,

$$
M=-\frac{64 I^{2} \sigma \epsilon b^{4}}{a \pi^{6}} \Sigma_{2}(b / a),
$$

which turns out to be negative. Moreover, a characteristic time $\tau$ of order $\epsilon$ can be identified as the ratio between mass and damping,

$$
\tau=|M| / \Gamma=\tau_{b} \frac{\Sigma_{2}(b / a)}{\Sigma_{1}(b / a)} .
$$

A similar calculation to get the damping coefficient and the effective mass may be carried out in frequency space. From Eq. (20), which gives the Fourier transform of the full response function, we can separate the real and the imaginary parts to get

$$
\hat{f}(\omega)=\frac{64 I^{2} \sigma}{a b^{2} \epsilon^{2}} \sum_{n, k=1}^{\infty} \frac{k^{2} \omega_{a}+n^{2} \omega_{b}-i \omega}{n^{2} \omega_{b}\left[\left(k^{2} \omega_{a}+n^{2} \omega_{b}\right)^{2}+\omega^{2}\right]},
$$

which allows us, by writing $\hat{f}(\omega)=\Gamma(\omega)-i \omega M$, to formally identify a frequency dependent damping coefficient,

$$
\Gamma(\omega)=\frac{64 I^{2} \sigma b^{2}}{a \pi^{4}} \sum_{n, k=1}^{\infty} \frac{k^{2}(a / b)^{2}+n^{2}}{n^{2}\left\{\left[k^{2}(a / b)^{2}+n^{2}\right]^{2}+\left(\omega / \omega_{b}\right)^{2}\right\}},
$$

and an effective mass,

$$
M(\omega)=-\frac{64 I^{2} \sigma \epsilon b^{4}}{a \pi^{6}} \sum_{n, k=1}^{\infty} \frac{1}{n^{2}\left\{\left[k^{2}(a / b)^{2}+n^{2}\right]^{2}+\left(\omega / \omega_{b}\right)^{2}\right\}} .
$$

Expanding to the first order in $\epsilon$,

$$
\hat{f}(\omega) \simeq \frac{64 I^{2} \sigma b^{2}}{a \pi^{4}} \Sigma_{1}(b / a)-i \omega \frac{64 I^{2} \sigma \epsilon b^{4}}{a \pi^{6}} \Sigma_{2}(b / a),
$$

we recover $\Gamma$ and $M$ as in Eqs. (25) and (26). 
Let us comment on the relevance of the eddy inertial term with respect to the usual term associated with the Döring mass $M_{D}$. The dynamics associated to the Döring mass $M_{D}$ is much faster than the one reported here, as it is a gyroscopic precessional effect. The corresponding mass, for a typical magnetic ribbon, is thus about 4 orders of magnitudes smaller than the eddy mass $M$. For the case of a magnetic ribbon with a thickness of around $10 \mu \mathrm{m}$, the eddy current mass is of order $M \sim 10^{-5} \mathrm{~kg} / \mathrm{m}^{2}$, while the Döring mass is of order $M_{D} \sim 10^{-9} \mathrm{~kg} / \mathrm{m}^{2}$. However, since $M$ depends on the third power of thickness (see the next section), we can estimate that the two masses become comparable in samples 1 or 2 orders of magnitude thinner, which is in the case of thick films.

\section{DEPENDENCE OF DAMPING AND MASS ON THE GEOMETRY OF THE SAMPLE}

Equations (26), (25), and (27) give the expression correct to the first order in $\epsilon$ of damping coefficient, effective mass, and characteristic time in a general geometry, defined by the parameters $a$ and $b$. The series $\Sigma_{1}$ and $\Sigma_{2}$ can be summed up for particular geometries. The calculations are reported in the Appendixes.

For a slab with $a \gg b$,

$$
\Sigma_{1}(b / a \rightarrow 0) \simeq \frac{a}{b} \frac{\pi}{4} \lambda_{3}, \quad \Sigma_{2}(b / a \rightarrow 0) \simeq \frac{a}{b} \frac{\pi}{8} \lambda_{5},
$$

where $\lambda_{n}=\bar{\Sigma}_{k} k^{-n}$, with $\lambda_{3}=1.05179 \ldots$ and $\lambda_{5}=1.00452 \ldots$, so that

$$
\Gamma=I^{2} \sigma \frac{16 \lambda_{3}}{\pi^{3}} b, \quad M=-I^{2} \sigma \epsilon \frac{8 \lambda_{5}}{\pi^{5}} b^{3}, \quad \tau=\epsilon \frac{\lambda_{5}}{2 \pi^{2} \lambda_{3}} b^{2} .
$$

For a slab with $a \ll b$,

$$
\Sigma_{1}(b / a \rightarrow \infty) \simeq \frac{\pi^{4}}{64} \frac{a^{2}}{b^{2}}, \quad \Sigma_{2}(b / a \rightarrow \infty) \simeq \frac{\pi^{6}}{768} \frac{a^{4}}{b^{4}},
$$

so that

$$
\Gamma=I^{2} \sigma a, \quad M=-I^{2} \sigma \epsilon \frac{a^{3}}{12}, \quad \tau=\epsilon \frac{a^{2}}{12} .
$$

For a square rod with $a=b$,

$$
\Sigma_{1}(1)=\frac{\pi^{4}}{128}, \quad \Sigma_{2}(1) \simeq 0.264 \pm 0.001,
$$

so that

$$
\Gamma=I^{2} \sigma_{2}^{\frac{b}{2}}, \quad M \simeq-I^{2} \sigma \epsilon 0.0175 b^{3}, \quad \tau \simeq \epsilon 0.0351 b^{2} .
$$

The results are summarized in Table I.

The dependence on $a(b)$ correctly disappears in the limit $a \rightarrow \infty(b \rightarrow \infty)$. The physical quantities $\Gamma, M$, and $\tau$ all increase with the overall sample size. For example, for a
TABLE I. Values of $\Gamma, M$, and $\tau$ for various geometries. $x$ denotes the smaller dimension between $a$ and $b$.

\begin{tabular}{lccc}
\hline \hline & $a \gg b$ & $a \ll b$ & $a=b$ \\
\hline$\Gamma /\left(I^{2} \sigma x\right)$ & 0.54 & 1 & 0.5 \\
$M /\left(I^{2} \sigma \epsilon x^{3}\right)$ & 0.026 & 0.083 & 0.0175 \\
$\tau /\left(\epsilon x^{2}\right)$ & 0.048 & 0.083 & 0.035 \\
\hline \hline
\end{tabular}

sample of a given thickness $a$, both damping coefficient and effective mass increase with $b$ and $b^{3}$, respectively. However, as soon as $b$ becomes larger than $a$, they saturate to a value proportional to $a$ and $a^{3}$, respectively. Since the mass increases faster than the damping with $b$, the characteristic time also increases with $b$ and saturates to a value proportional to $a^{2}$. A similar behavior is observed by varying $a$ at fixed $b$, although the role of the two dimensions transverse and parallel to the wall is not symmetric. Essentially, the dependence of $\Gamma, M$, and $\tau$ on the geometry of the sample is dominated by the smaller sample dimension between $a$ and $b$. Thus, the relevance of the eddy current dynamic effect is controlled by the smaller sample dimension: the thinner the sample, the smaller the effect, while the rest of the geometry does not play any relevant role. The characteristic time in $\epsilon$ units is approximately equal to $\tau / \epsilon \simeq 0.083 a^{2}$ when $a<b$, $\tau / \epsilon \simeq 0.048 b^{2}$ when $b<a$, and has its minimum $\tau / \epsilon$ $\simeq 0.035 b^{2}$ for a square rod. In all cases, it stays between $3 \%$ and $10 \%$ of the squared relevant sample size.

Figures 2-4 show the behavior of $\Gamma, M$, and $\tau$ both as a function of parallel dimension $b$, for $a=1$, and of the transverse dimension $a$, for $b=1$. The straight lines are fits with the asymptotic behaviors calculated for the large $a$ and $b$ limits.

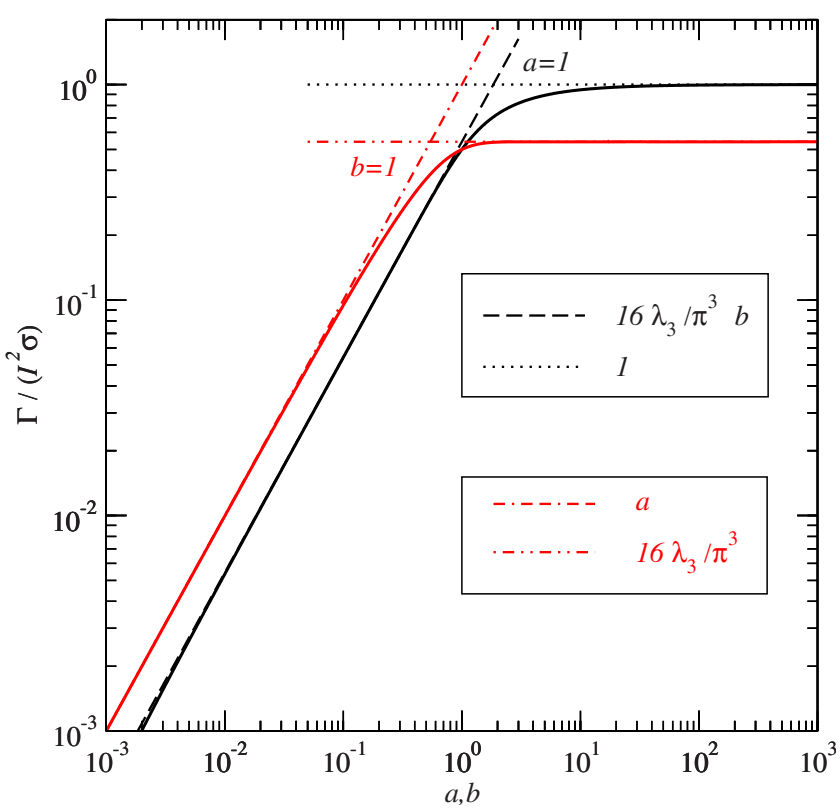

FIG. 2. (Color online) Log-log plot of the damping coefficient $\Gamma$ in $I^{2} \sigma$ units as a function of $b$ for $a=1$ (black line) and as a function of $a$ for $b=1$ [red (dark gray) line]. The fits correspond to the analytic calculation. 


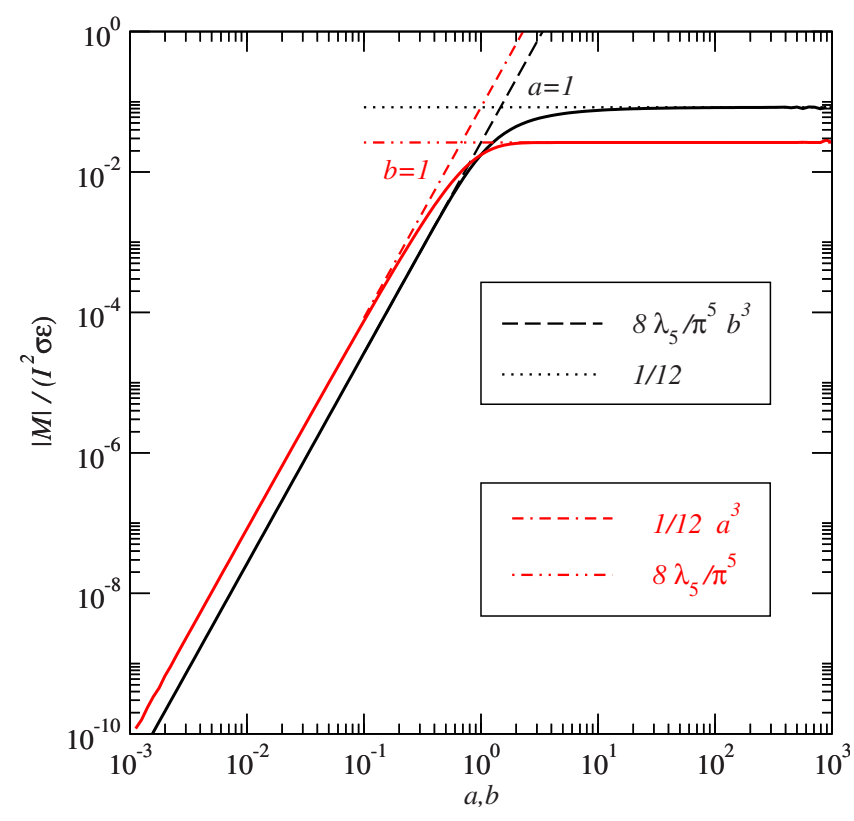

FIG. 3. (Color online) Log-log plot of the absolute value of the effective mass $M$ in $I^{2} \sigma \epsilon$ units as a function of $b$ for $a=1$ (black line) and as a function of $a$ for $b=1$ [red (dark gray) line]. The fits correspond to the analytic calculation.

\section{COMMENTS AND CONCLUSIONS}

The asymmetry in the Barkhausen pulses is due to the noninstantaneous response of the field to the domain wall displacement. Most treatments of the Barkhausen noise assume the eddy current drag on the magnetic domain wall to

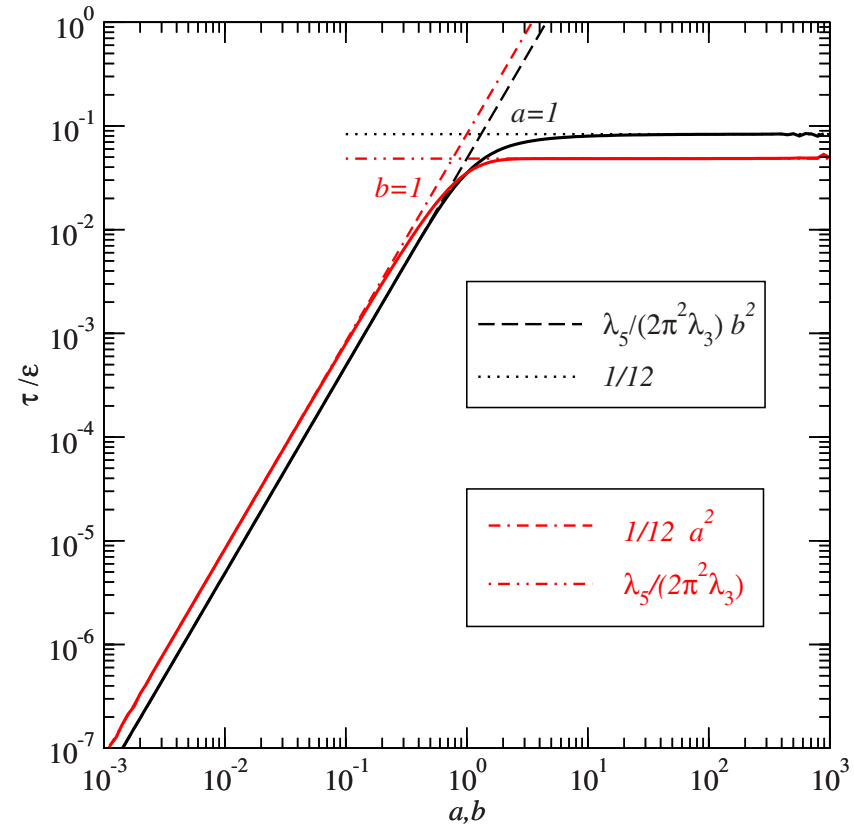

FIG. 4. (Color online) Log-log plot of the characteristic time $\tau$ in $\epsilon$ units as a function of $b$ for $a=1$ (black line) and as a function of $a$ for $b=1$ [red (dark gray) line]. The fits correspond to the analytic calculation. be instantaneous, which corresponds to assuming $\epsilon=\sigma \mu=0$ within the magnetic domains. However, eddy currents take a finite time to set up after the magnetic reversal, and persist for a finite time after the corresponding wall displacement. This time delay causes the eddy pressure on the wall at a given time $t$ not to be strictly proportional to the instantaneous velocity of the wall but to depend on a weighted average of velocities of the wall up to time $t$. This quasi-inertial effect is responsible for the asymmetry in the pulse shape observed in the Barkhausen experiments. The delay has a characteristic timescale $\tau$, and therefore, its effect is more severe on avalanches of comparable duration. For very long avalanches, the separation of time scales is such that the response of the field can be assumed to be instantaneous; therefore, the asymmetry disappears, and strict universality is recovered.

Starting from the full Maxwell equation, which includes the dynamic eddy current effects, we calculate the retarded pressure on the wall. The first order correction to the quasistatic $(\epsilon=0)$ solution leads us to identify a damping coefficient, which survives to the $\epsilon \rightarrow 0$ limit and coincides with the one calculated assuming an instantaneous response, and an effective mass, which vanishes in the $\epsilon \rightarrow 0$ limit. A damping coefficient and an effective mass can be formally defined beyond the first order approximation; however, they both result to be frequency dependent. Damping coefficient, effective mass, and characteristic time depend on the sample geometry; however, it turns out that the only geometrical parameter that significantly affects them is the smaller sample dimension.

The effective mass results to be negative at all frequencies. The leftward pulse asymmetry observed in the Barkhausen experiments is indeed consistent with a negative effective mass: the avalanche starts fast and end slowly, which is exactly the opposite of what one would expect from standard inertia. This may be understood by observing that the retarded pressure at time $t$ is proportional to an average of previous velocities of the wall up to time $t$. When the wall is accelerating, the effective average velocity is smaller than the instantaneous one, thus, the wall experiences less retardation than it would if it was moving at constant velocity, and the drag $\beta v$ has to be corrected with a negative term $M d v / d t$ to account for this. The opposite is true when the wall decelerates. However, to understand the physical meaning of the negative mass, one has always to keep in mind that the inertial term has been introduced as a convenient way to account, to the first order, for the history dependent damping on the wall. Therefore, it cannot describe, for example, the very short time dynamics of a wall starting to move from rest: in this case, a negative mass would cause the wall to move backward. However, since when the wall starts to move, there is no previous history of motion, both eddy damping and eddy inertial terms are zero, and the short time dynamics is governed instead by the positive Döring mass.

\section{APPENDIX A: SUM OF THE SERIES $\Sigma_{1}(\alpha)$ FOR $\alpha=1$ AND IN THE LIMIT $\alpha \rightarrow \infty, \alpha \rightarrow 0$}

We here calculate the sum of the series, 


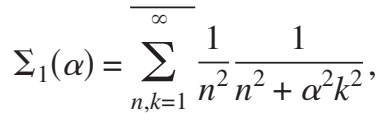

for some specific values of $\alpha$. The value of $\Sigma_{1}$ for $\alpha=1$ can be obtained by writing a closed equation for $\Sigma_{1}(1)$ as follows:

$$
\begin{aligned}
\sum_{n, k=1}^{\infty} \frac{1}{n^{2}} \frac{1}{n^{2}+k^{2}} & =\sum_{n, k=1}^{\infty}\left(\frac{1}{n^{2}}-\frac{1}{n^{2}+k^{2}}\right) \frac{1}{k^{2}} \\
& =\sum_{n, k=1}^{\infty} \frac{1}{n^{2}} \frac{1}{k^{2}}-\sum_{n, k=1}^{\infty} \frac{1}{k^{2}} \frac{1}{n^{2}+k^{2}},
\end{aligned}
$$

which gives

$$
2 \sum_{n, k=1}^{\infty} \frac{1}{n^{2}} \frac{1}{n^{2}+k^{2}}=\left(\overline{\sum_{n, k=1}^{\infty} \frac{1}{k^{2}}}\right)^{2}=\left(\frac{\pi^{2}}{8}\right)^{2}
$$

so that

$$
\Sigma_{1}(1)=\pi^{4} / 128
$$

To calculate $\Sigma_{1}(\alpha)$ in the limit $\alpha \rightarrow 0$, we use Eq. (18) with $z=n \pi / 2 \alpha$ to write

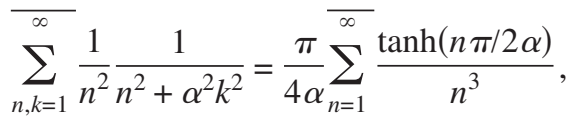

which, in the limit $\alpha \rightarrow 0$, gives

$$
\Sigma_{1}(\alpha \rightarrow 0) \simeq \frac{\pi}{4 \alpha} \lambda_{3}
$$

In the opposite limit $\alpha \rightarrow \infty$, using again Eq. (A5) and the sum of $\lambda_{n}=\bar{\Sigma}_{k} k^{-2}=\pi^{2} / 8$, we get

$$
\Sigma_{1}(\alpha \rightarrow 0) \simeq \frac{\pi^{4}}{64 \alpha^{2}}
$$

\section{APPENDIX B: SUM OF THE SERIES $\Sigma_{2}(\alpha)$ IN THE LIMIT $\alpha \rightarrow \infty, \alpha \rightarrow 0$}

We here calculate the sum of the series,

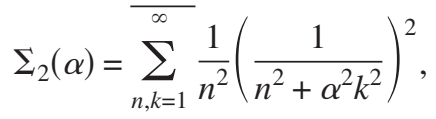

for some specific values of $\alpha$. To calculate $\Sigma_{1}(\alpha)$ in the limit $\alpha \rightarrow 0$, let us write $\Sigma_{2}$ as

$$
\Sigma_{2}(\alpha)=\lim _{\epsilon \rightarrow 0} \frac{1}{\sum_{n, k=1}^{\infty}} \frac{1}{n^{2}}\left(\frac{1}{n^{2}+\alpha^{2} k^{2}}-\frac{1}{n^{2}+\alpha^{2} k^{2}+\epsilon}\right),
$$

and then use Eq. (18) in both terms with $z=n \pi / 2 \alpha$ and $z=\sqrt{n^{2}+\epsilon} \pi / 2 \alpha$, respectively,

$$
\begin{aligned}
\Sigma_{2}(\alpha)= & \lim _{\epsilon \rightarrow 0} \frac{1}{\epsilon} \sum_{n}^{\infty} \frac{1}{n^{2}} \frac{\pi}{4 \alpha}\left(\frac{\tanh (\pi n / 2 \alpha)}{n}\right. \\
& \left.-\frac{\tanh \left(\pi \sqrt{n^{2}+\epsilon} / 2 \alpha\right)}{\sqrt{n^{2}+\epsilon}}\right) .
\end{aligned}
$$

Expanding for small $\epsilon$ and taking the limit, one gets

$$
\Sigma_{2}(\alpha)=\frac{\pi}{8 \alpha} \sum_{n}^{\infty} \frac{\tanh (\pi n / 2 \alpha)}{n^{5}}-\frac{\pi^{2}}{16 \alpha^{2}} \sum_{n}^{\infty} \frac{1-\tanh ^{2}(\pi n / 2 \alpha)}{n^{4}} .
$$

Now, we can finally take the limit $\alpha \rightarrow 0$ to get

$$
\Sigma_{2}(\alpha \rightarrow 0) \simeq \frac{\pi}{8 \alpha} \sum_{n}^{\infty} \frac{1}{n^{5}}=\frac{\pi \lambda_{5}}{8 \alpha} .
$$

The limit $\alpha \rightarrow \infty$ can also be obtained from Eq. (B4) by using the expansion $\tanh (x) \simeq x-x^{3} / 3$ for small arguments, which gives

$$
\begin{aligned}
\Sigma_{2}(\alpha \rightarrow \infty) & \simeq \frac{\pi}{8 \alpha} \sum_{n}^{\infty}\left(\frac{\pi}{2 \alpha} \frac{1}{n^{4}}-\frac{\pi^{3}}{3(8 \alpha)^{3}} \frac{1}{n^{2}}\right) \\
& -\frac{\pi^{2}}{16 \alpha^{2}} \sum_{n}^{\infty}\left(\frac{1}{n^{4}}-\frac{\pi^{2}}{(2 \alpha)^{2}} \frac{1}{n^{2}}\right)=\frac{\pi^{6}}{768 \alpha^{4}} .
\end{aligned}
$$

${ }^{1}$ G. Durin and S. Zapperi, in The Science of Hysteresis, edited by G. Bertotti and I. Mayergoyz (Academic, New York, 2005), p. 181267.

${ }^{2}$ G. Bertotti, Hysteresis in Magnetism (Academic, San Diego, 1998).

${ }^{3}$ B. Alessandro, C. Beatrice, G. Bertotti, and A. Montorsi, J. Appl. Phys. 68, 2901 (1990).

${ }^{4}$ S. Zapperi, P. Cizeau, G. Durin, and H. E. Stanley, Phys. Rev. B 58, 6353 (1998).

${ }^{5}$ K. P. O'Brien and M. B. Weissman, Phys. Rev. E 50, 3446 (1994)

${ }^{6}$ J. Sethna, K. A. Dahmen, and C. R. Myers, Nature (London) 410, $242(2001)$
${ }^{7}$ D. Spasojevic, S. Bukvic, S. Milosevic, and H. E. Stanley, Phys. Rev. E 54, 2531 (1996).

${ }^{8}$ A. P. Mehta, A. C. Mills, K. Dahmen, and J. P. Sethna, Phys. Rev. E 65, 046139 (2002).

${ }^{9}$ F. Colaiori, G. Durin, and S. Zapperi, J. Magn. Magn. Mater. 272, E533 (2004).

${ }^{10}$ S. Zapperi, C. Castellano, F. Colaiori, and G. Durin, Nat. Phys. 1, 46 (2005).

${ }^{11}$ J. E. L. Bishop, J. Phys. D 13, L15 (1980).

${ }^{12}$ The definition of magnetic permeability in the Maxwell equation for the eddy field needs a clarification: unlike the case of an ideal homogeneous material, in the case of irreversible domain wall propagation, there is no clear definition of permeability of 
the medium. In principle, one should use the permeability of the medium where eddy currents occur; therefore, neither the vacuum permeability nor the reversible permeability is appropriate, since the process we deal with involves a real soft magnet during the irreversible domain wall displacement and far from saturation. In this case, the value which better represents the permeability of the medium is rather the average linear permeability of the hysteresis loop around the coercive field, which indeed properly takes into account the multidomain configuration, the intrinsic damping, and the average effect of the eddy currents. 